\title{
Criterios \\ Fundamentos y antecedentes de la utilización de enzimas en tratamientos de limpieza
}

Isabel Blasco Castiñeyra > Sonsoles de la Viña Ferrer > Margarita San Andrés Moya

Dpto. de Pintura-Restauración. Universidad Complutense de Madrid

\section{Resumen}

Se plantean las posibilidades de empleo de las enzimas en tratamientos de limpieza, como alternativa a los métodos tradicionales. Se describen todos los aspectos que hay que considerar para su utilización: tipo de enzimas, estructura, forma de actuación y condiciones de trabajo. Asimismo, se revisan las publicaciones relacionadas con el empleo de este tipo de sistemas en tratamientos de restauración, en las que se discuten su forma de aplicación así como las condiciones que hay que tener en cuenta para su uso.

\section{Introduccion}

Dentro del contexto de la conservación y restauración de obras de arte, el proceso de limpieza de las superficies policromadas es uno de los más importantes y delicados. La suciedad acumulada, el deterioro y oscurecimiento de los barnices, así como la presencia de repintes $u$ otras materias ajenas a la obra, pueden provocar variaciones notables en su aspecto estético original. Estas alteraciones, además de causar importantes modificaciones en su lectura e interpretación, en ocasiones pueden suponer un factor de deterioro adicional para la obra de arte.

Así, el principal objetivo de las operaciones de limpieza es la eliminación selectiva de materiales degradados, suciedad y repintes, mediante la utilización de métodos mecánicos o físico-químicos, sin que se vean afectados los componentes originales de la obra.

Las sustancias utilizadas tradicionalmente en la limpieza de obras de arte se pueden agrupar en cinco categorías: abrasivos, reactivos alcalinos, tensoactivos, agentes quelantes y, fundamentalmente, disolventes orgánicos. Desde la antigüedad clásica y hasta el siglo XVIII los procedimientos de limpieza se basaron principalmente en la utilización de reactivos alcalinos, jabones y abrasivos (Caley, 1990). Los disolventes fueron utilizados de forma más frecuente a partir del siglo XIX, después de la introducción de los barnices a la esencia (Masschelein-Kleiner, 1991); mientras que los tensoactivos, junto con los agentes quelantes, son métodos más recientes (Wolbers 2000: 27-126).

En general, los sistemas citados como tradicionales presentan dos problemas fundamentales: la escasa especificidad en su acción y la elevada retención que algunas de las sustancias empleadas puedan tener en la superficie de la obra de arte.

La utilización de medios alcalinos presenta como principal inconveniente sus valores de $\mathrm{pH}$. A este respecto, las soluciones con un valor de $\mathrm{pH}$ superior a 8,5 pueden llegar a provocar la ruptura de la pelicula pictórica oleosa, mediante un proceso de saponificación ${ }^{1}$ de los aceites (Wolbers, 1990:101) y dar lugar a una pérdida irreversible de su poder de cohesión. Otra consecuencia del tratamiento con disoluciones alcalinas es el efecto conocido como blanqueamiento ${ }^{2}$ de la policromía y que, según Burnstock y Learner (1992), podría deberse a la pérdida de cohesión entre el pigmento y el aglutinante.

Diversos autores han estudiado los efectos que los disolventes orgánicos pueden provocar sobre las capas pictóricas (Feller et al., 1985; Stolow, 1957a y 1957b; Phenix and Sutherland, 2001); entre estos efectos se podrían citar los siguientes:

$>$ Los fenómenos de lixiviación desarrollados sobre las películas de óleo envejecidas provocan la eliminación de componentes no polimerizados y de moléculas de pequeño tamaño formadas durante el 


\footnotetext{
${ }^{1}$ Hidrólisis del enlace ester que conduce a la formación de un alcohol y la sal del ácido correspondiente

2 "Blanching" en inglés y "Blanchiment" en francés

${ }^{3}$ También conocidos como agentes secuestrantes

${ }^{4}$ Desfloculación: proceso de ruptura de agregados de partículas en unidades más pequeñas. En este caso la adsorción del quelante, que presenta carga negativa a determinados valores de $\mathrm{pH}$, hace que las partículas se separen por repulsión de las cargas del mismo signo
}

proceso de secado y envejecimiento, los cuales actúan como plastificantes (Erhardt and Tsang, 1990). Como consecuencia, se produce una disminución de la elasticidad de la película pictórica con el consiguiente aumento de su dureza y fragilidad (Hedley et al., 1990).

$>$ El proceso de hinchamiento provocado por el propio mecanismo de actuación de los disolventes orgánicos hace que el aglutinante experimente un aumento de volumen, que a su vez puede originar una compresión lateral de la película y dar lugar a una posible alteración de tipo mecánico (Feller et al., 1985:54).

$>$ Algunos pigmentos pueden presentar mayor afinidad por los disolventes utilizados en la limpieza que por el propio medio con el que están aglutinados.

> Otro problema importante es la toxicidad de los disolventes orgánicos para el propio restaurador (Marchi e Ottogalli, 1999) y el efecto contaminante que ejercen sobre el medio ambiente.

Los tensoactivos, de introducción más reciente en el campo de la restauración, pueden ocasionar problemas por su elevada retención en la obra y, en algunos casos, por su posible transformación en sustancias muy reactivas, como son los peróxidos. Algunos autores consideran que estos peróxidos pueden potenciar el inicio de reacciones de degradación, tras su descomposición en radicales libres (Burnstock and White, 1990).

Los agentes quelantes ${ }^{3}$, sustancias capaces de formar complejos solubles en agua con ciertos iones metálicos, se han utilizado como agentes de limpieza de superficies policromadas (Carlyle et al., 1990; Phenix and Burnstock, 1992; Wolbers, 2000:109126). Entre los más empleados se encuentran las sales del ácido cítrico (citrato de diamonio y citrato de triamonio) y el ácido etilendiamino tetracético (EDTA). Sin embargo, el mecanismo de acción de estas sustancias parece que no se debe exclusivamente a su comportamiento como agente secuestrante. Según Phenix y Burnstock (1992) también ejercen mecanismos de adsorción, desfloculación ${ }^{4}$ y dispersión de las partículas de suciedad, además de tener cierto carácter tensoactivo. Por este motivo, existe un riesgo de que estos compuestos puedan dañar las películas de óleo. Por otra parte, ciertos agentes quelantes presentan una gran afinidad por determinados iones; por ejemplo, el citrato forma complejos muy estables con los iones $\mathrm{Fe}^{3+}$ y $\mathrm{Cu}^{2+}$. Por tanto, las peliculas con pigmentos ricos en estos iones, como ocres y tierras (ricos en $\mathrm{Fe}^{3+}$ ) o malaquita y azurita (ricos en $\mathrm{Cu}^{2+}$ ), serán más vulnerables a la acción de este agente quelante (Phenix and Burnstock, 1992).

Los problemas descritos han llevado a la búsqueda de sistemas de limpieza alternativos más especificos y que provoquen el menor daño posible sobre las obras de arte. Las tendencias actuales están, por tanto, orientadas al diseño de nuevas metodologías centradas en la utilización de sistemas menos agresivos y más específicos, que actúen únicamente sobre los materiales que se van a eliminar.

Dentro de este contexto, surge el planteamiento de la utilización de enzimas como alternativa a los procesos de limpieza tradicionales, en aquellos casos en los que éstos no resulten eficaces o se quiera evitar su acción inespecífica.

\section{Enzimas. Conceptos generales}

Antes de profundizar en los aspectos relacionados con la utilización de enzimas en procesos de restauración, es conveniente revisar algunos conceptos generales relacionados con la estructura de estas macromoléculas, su nomenclatura y tipos.

Las enzimas son proteinas sintetizadas en las células vivas que tienen la particularidad de presentar actividad catalítica. Como consecuencia de esta propiedad, pueden aumentar la velocidad de ciertas reacciones químicas de transformación de una sustancia, designada sustrato, sin sufrir ellas mismas cambios permanentes en su estructura. Esta acción se consigue mediante la disminución de la barrera energética necesaria para que estas reacciones se desarrollen; este efecto se logra a través de la formación de un complejo intermedio enzima-sustrato, que posteriormente se transforma en el producto de la reacción, recuperándose nuevamente la enzima libre (Lehninger, 1985:195; Fersht, 1985:25-26).

Las proteinas son largas cadenas de aminoácidos unidos a través de enlaces covalentes. Se conocen algo más de veinte aminoácidos distintos, que tienen en común un átomo de carbono (llamado carbono alpha; $\mathrm{C}_{\alpha}$ ) al cual se unen: un grupo carboxilo $(\mathrm{COOH})$, un grupo amino $\left(\mathrm{NH}_{2}\right)$ y un átomo de hidrógeno; la cuarta valencia del carbono está unida a un radical o cadena lateral, cuya naturaleza determina a su vez la del aminoácido. Por tanto, los distintos aminoácidos se diferencian en la estructura de su cadena lateral, que puede ser de naturaleza hidrofóbica (apolar), polar o incluso presentar carga a determinados valores de $\mathrm{pH}$. Las proteínas son, por tanto, polielectrolitos ya que poseen grupos funcionales ionizables que son de distinta naturaleza según el $\mathrm{pH}$ del medio. El grupo amino se encuentra ionizado en forma catiónica a pH ácido $\left(\mathrm{R}-\mathrm{NH}_{2}\right.$ $+\mathrm{H}^{+} \rightrightarrows \mathrm{R}-\mathrm{NH}_{3}{ }^{+}$), mientras que el grupo carboxilico está ionizado en forma aniónica a pH alcalino ( $\left.\mathrm{R}-\mathrm{COOH}+\mathrm{OH} \Rightarrow \mathrm{R}-\mathrm{COO}+\mathrm{H}_{2} \mathrm{O}\right)$.

En el proceso de sintesis de las proteinas, los aminoácidos van uniéndose a través de enlaces peptídicos, que se forman mediante una reacción de condensación que tiene lugar entre el grupo carboxilico de un aminoácido y el grupo amino del siguiente, eliminándose una molécula de agua (Fig.1).

La secuencia concreta de aminoácidos constituye la denominada estructura primaria de la proteína. La naturaleza de estos aminoácidos 


\section{Criterios \\ Fundamentos y \\ antecedentes de la \\ utilización de enzimas en \\ tratamientos de limpieza \\ Isabel Blasco Castiñeyra \\ y otros}

hace que las proteinas adquieran una disposición espacial concreta (helicoidal, plegamiento B, estructura al azar), que a su vez puede plegarse en una estructura tridimensional característica en el entorno celular en el que se localizan; esta estructura tridimensional se denomina conformación nativa de la proteína y está estabilizada a través de la formación de interacciones de diversa naturaleza: no covalentes (electrostáticas, puentes de hidrógeno, hidrofóbicas) o covalentes (puentes disulfuro). Como consecuencia de esta estructura, las proteínas son muy sensibles a los cambios que se producen en su entorno, es decir, temperatura, $\mathrm{pH}$ y la presencia de disolventes o de sustancias iónicas. Una variación en estos parámetros puede modificar las interacciones que la estabilizan y dar lugar a la pérdida de su conformación nativa en un proceso denominado desnaturalización. Esta desnaturalización provoca una pérdida de la funcionalidad de la proteina, que en el caso concreto de las enzimas se traduce en una pérdida de su actividad catalítica.

Generalmente, las enzimas se clasifican teniendo en cuenta el tipo de reacción que catalizan, y se nombran añadiendo el sufijo "asa" al nombre que hace referencia a la reacción catalizada. De esta forma se habla de:

> Transferasas: catalizan reacciones de transferencia de ciertos grupos funcionales.

> Oxidoreductasas: catalizan reacciones de oxidación-reducción.

$>$ Hidrolasas y liasas ${ }^{5}$ : catalizan la ruptura de enlaces químicos.

$>$ Ligasas: catalizan la formación de nuevos enlaces.

> Isomerasas: catalizan la conversión de la molécula en una estructura espacial determinada.

Una clasificación más completa debe considerar el tipo de sustrato sobre el que se produce la transformación. Dada la complejidad de este tema y para evitar confusiones, existe una comisión internacional, la Comisión de Enzimas (EC) de la Unión Internacional de Bioquímica, que regula su nomenclatura. Según esta Comisión, cada enzima viene caracterizada por un subíndice de cuatro cifras $\left(E C_{x . x . x . x}\right)$, cada una de las cuales indica, respectivamente: clase 0 tipo de reacción ( $1=$ oxidoreductasa, $2=$ transferasa, $3=$ hidrolasa, $4=$ liasa, $5=$ isomerasa, $6=$ ligasa), subclase 0 grupo funcional que es modificado, aspectos específicos de la reacción y, por último, el número progresivo de las enzimas que pertenecen a ese grupo.

En el campo de la restauración, y concretamente en el caso de los tratamientos de limpieza de obras de arte, las enzimas que presentan mayor interés son las hidrolasas (grupo $n^{\circ} 3$ ), ya que mediante la hidrólisis selectiva de ciertas sustancias, se puede lograr la eliminación de aquellos materiales que alteran la obra y se han vuelto irreversibles con el paso del tiempo. Dentro de las hidrolasas, existen diferentes subclases dependiendo del grupo funcional que es hidrolizado:

$>$ Enzimas lipolíticas $\left(\mathrm{EC}_{31 \mathrm{x}}\right)$ : enzimas digestivas indispensables para el metabolismo de las sustancias grasas. Dentro de este
${ }^{5}$ Hidrolasas: catalizan reacciones de hidrólisis (ruptura de enlaces) Liasas: catalizan reacciones de eliminación con la formación de un doble enlace o la adición de grupos a dobles enlaces

${ }^{6}$ La unión glicosídica de la celulosa es B1-4. Estos enlaces son hidrolizados por la celulasa $\left(\mathrm{EC}_{3.2 .1 .4}\right)$

${ }^{7}$ Hidrólisis de los enlaces peptídicos de la propia enzima

grupo son de especial interés las lipasas $\left(\mathrm{EC}_{3.1 .1 .3}\right)$ que catalizan la hidrólisis de los triacilglicéridos presentes en el aceite de lino, descomponiéndolos en glicerol y sus correspondientes ácidos grasos.

$>$ Glicosidasas $\left(\mathrm{EC}_{3.2 . x . x}\right)$ : enzimas digestivas que permiten la degradación de los carbohidratos. Dentro de este grupo son de especial interés las $\alpha$-amilasas $\left(\mathrm{EC}_{3211}\right)$, que son capaces de hidrolizar los enlaces glicosídicos $\alpha$ 1-4 del almidón, presentes en la harina. Debido a su especificidad, estas enzimas no tienen efecto sobre la celulosa ${ }^{6}$, principal componente del papel y la madera.

$>$ Enzimas proteolíticas $\left(\mathrm{EC}_{3.4 . \mathrm{x.x}}\right)$ : son enzimas capaces de degradar proteinas como la gelatina, colas animales, caseina y proteinas del huevo. Algunas enzimas proteoliticas presentan una gran especificidad de sustrato, siendo capaces de reconocer e hidrolizar sólo aquellos enlaces adyacentes a ciertos aminoácidos. Las enzimas proteolíticas presentan la particularidad de que, en ausencia de sustrato, pueden degradarse en un proceso conocido como autolísis ${ }^{7}$.

Por tanto, dependiendo del tipo de sustancias que se quieran eliminar en la obra, las enzimas utilizadas serán proteasas, lipasas o amilasas, las cuales fragmentarán, respectivamente, proteínas, lípidos 0 almidón.

Aplicación práctica de las enzimas, propiedades a considerar Dentro de las propiedades caracteristicas de las enzimas, hay que destacar su especificidad, eficacia catalítica y estabilidad. Todas ellas están relacionadas con su actividad catalítica y vienen determinadas, no sólo por el tipo de enzima, sino también por la naturaleza del sustrato y las condiciones del medio en que se encuentran.

Una de las propiedades más singulares de las enzimas es la especificidad que presentan por ciertos sustratos, lo cual supone una gran ventaja para su uso en procesos de restauración de obras de arte. Las enzimas están intrínsecamente dotadas de selectividad, no sólo por el tipo de sustancia que transforman, el sustrato, sino también por el tipo de reacción que catalizan.

La formación del complejo enzima-sustrato tiene lugar en una pequeña región de la enzima denominada centro activo (Price and Stevens, 1989:139), el cual suele estar localizado en un hueco o cavidad de su propia estructura proteica. La especificidad enzimática reside inherentemente en este centro activo, que posee las dimensiones correctas, así como la topología y naturaleza adecuadas para acomodarse a un sustrato específico. Además, dentro de este centro activo se encuentran también los grupos funcionales implicados en la reacción catalítica.

Algunas enzimas poseen una especificidad casi absoluta con respecto a un sustrato determinado y no actúan sobre otras moléculas aunque estén muy relacionadas estructuralmente; por el con- 
Figura 1. estructura de un aminoácido y enlace peptídico

Figura 2. Modelo de encaje inducido (adaptado de Lehninger, 1985). Enzima

libre con centro activo en forma relajada y cómo la unión del sustrato hace que

la enzima se acople al mismo.

Gráfico 1. Variación de la actividad enzimática según el pH del medio.<smiles>[R]C(N)C(=O)O</smiles><smiles>[R]C(N)C(=O)OCCNC([R1])C(=O)O</smiles><smiles>[R7]C(N)C(=O)CCCC</smiles><smiles>[R]C(N)C(=O)O</smiles>

enlace peptídico
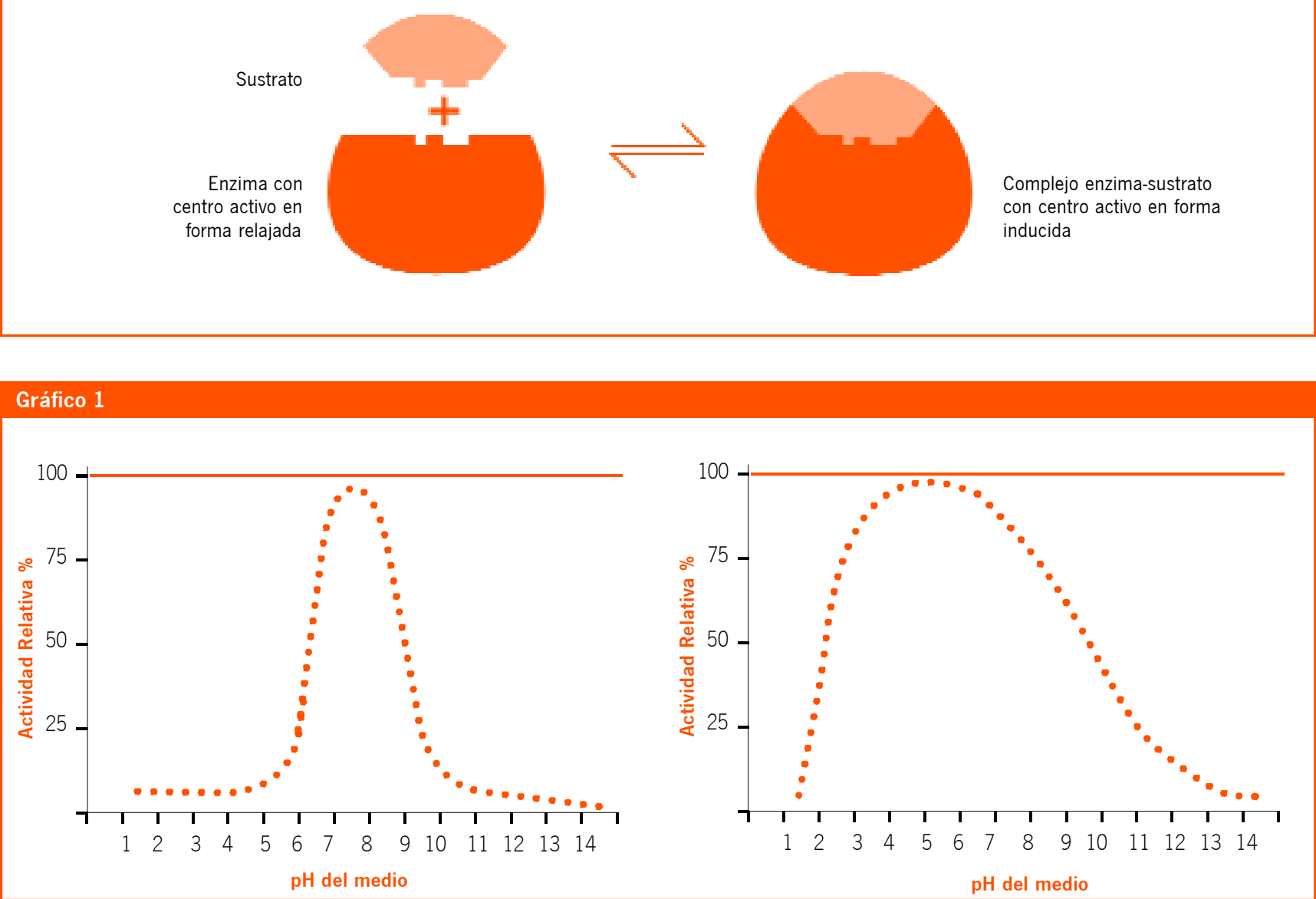


\section{Criterios \\ Fundamentos y \\ antecedentes de la \\ utilización de enzimas en \\ tratamientos de limpieza \\ Isabel Blasco Castiñeyra \\ y otros}

trario, otras enzimas actúan sobre todo un mismo tipo de moléculas con un común denominador estructural, aunque lo hagan a velocidades diferentes (Lehninger, 1985:224).

Se han propuesto varios modelos para explicar la selectividad de las enzimas sobre un determinado sustrato y su modo de actuación. El modelo inicial de la llave y cerradura, en el que la enzima y el sustrato eran consideradas estructuras estáticas complementarias (Fischer, 1894), ha sido ampliamente superado por el modelo del acoplamiento o encaje inducido (Koshland and Neet, 1968). Según este último, los grupos funcionales esenciales, situados en el centro activo de la enzima libre, no se hallan en sus posiciones óptimas para promover la unión del sustrato a la enzima cuando dicho centro activo no está ocupado. Sin embargo, la unión del sustrato a la enzima obliga a ésta a adoptar una conformación favorable, en la que los grupos catalíticos pueden iniciar la reacción; es decir, se produce un encaje o ajuste inducido. En esta conformación activa la molécula de enzima es inestable y, por tanto, en ausencia de sustrato, tiende a recuperar su conformación nativa original (Lehninger 85:236) (Figura 2).

Esta especificidad de sustrato hace posible que existan enzimas con capacidad para distinguir entre diferentes tipos de proteínas. Esta propiedad permite que en una misma obra se pueda llevar a cabo la eliminación selectiva de ciertos materiales proteicos, como por ejemplo un aglutinante de cola, sin afectar una capa pictórica ejecutada con temple de huevo. Esta característica supone una gran ventaja en cuanto a las necesidades de selectividad en los procesos de limpieza de obras de arte, y no se consigue con los métodos tradicionales citados anteriormente.

Otra propiedad interesante de las enzimas es su eficacia. A este respecto, las enzimas catalizan las reacciones con una mayor eficacia que los catalizadores inorgánicos. Como ejemplo tenemos la reacción de descomposición del agua oxigenada en agua y oxigeno. En ausencia de catalizador, la energía de activación necesaria para que suceda esta reacción es de $75 \mathrm{~kJ} / \mathrm{mol}$. En cambio, la energía es de $50 \mathrm{~kJ} / \mathrm{mol}$ en presencia de platino (catalizador inorgánico) y disminuye hasta $8 \mathrm{~kJ} / \mathrm{mol}$ en presencia de una enzima como la peroxidasa. Otra ventaja de las enzimas, respecto a los catalizadores inorgánicos, es que, aunque las enzimas son proteinas y por tanto moléculas relativamente frágiles, son capaces de desarrollar sus extraordinarios efectos cataliticos en disoluciones acuosas diluidas, a pH biológico y a temperatura moderada.

Respecto a su estabilidad, las enzimas se pueden aislar de la estructura celular de la que proceden y pueden actuar independientemente de ésta, tanto sobre sustratos naturales como sintéticos; esta circunstancia ha permitido su utilización fuera del entorno celular, de forma controlada y no como parte del proceso asociado al biodeterioro. Sin embargo, en estas condiciones, las enzimas sufren un proceso progresivo de degradación y pueden perder su funcionalidad ya que, como se ha mencionado, la conformación tridimensional es imprescindible para que desempeñen su función. Por este motivo las enzimas tienen que ser utilizadas en unas condiciones semejantes a las del medio celular del que proceden. Hay que resaltar que estas condiciones de trabajo, además de ser necesarias para que se mantenga la actividad enzimática, son en sí mismas poco agresivas para el tratamiento de obras policromadas, a diferencia de lo que ocurre con otros procedimientos tradicionales de limpieza.

En cuanto a su toxicidad, aunque las proteasas, lipasas y amilasas están clasificadas como sustancias poco tóxicas, durante su manipulación conviene tomar ciertas precauciones. Concretamente, si se encuentran en forma de polvo, es necesario evitar su inhalación y el contacto con la piel. Sin embargo, una vez preparada la correspondiente disolución acuosa para proceder a su aplicación, el uso de guantes de látex ofrece una protección suficiente, lo cual representa una ventaja adicional para su empleo (Cremonesi, 1997:107).

\section{La utilización de enzimas en la limpieza de policromías. Factores implicados y condiciones operativas}

Para poder entender el mecanismo de acción de las enzimas en tratamientos de limpieza, hay que considerar la obra de arte policromada como una superposición de estratos, constituidos por sustancias de diversa naturaleza química. Estas sustancias son, fundamentalmente, las proteínas, los lípidos y los polisacáridos, principales compuestos orgánicos de origen natural utilizados como aglutinantes en las técnicas pictóricas tradicionales y también como adhesivos en numerosos procesos de restauración.

Las proteinas se encuentran principalmente en la técnica del temple de huevo, en los barnices de clara de huevo y en la cola de origen animal que es utilizada, por ejemplo, en la aplicación de las capas de preparación y en ciertos procesos de restauración.

Los lípidos forman parte de la composición de los aceites secantes empleados en la técnica del óleo y también son lípidos las ceras de origen animal y vegetal, utilizadas como recubrimiento o adhesivo y como aglutinante en la técnica de la encaústica.

Los polisacáridos intervienen en la composición de materiales tan diversos como los derivados de la celulosa, las gomas polisacáridas de origen vegetal, empleadas en técnicas como la acuarela y en los adhesivos como el almidón.

Todas estas sustancias forman películas con ciertas propiedades fisico-químicas (adhesividad, flexibilidad, solubilidad...), que dependerán tanto de su composición inicial como de aquellos procesos desarrollados durante su endurecimiento y posterior envejeci- 
miento, que en mayor o menor grado afectarán a su estructura química original.

Aunque sólo los polisacáridos y las proteinas son sustancias inicialmente poliméricas, de elevado tamaño molecular, en el caso de los aceites secantes, los triacilglicéridos poliinsaaturados que forman parte de su composición se van entrecruzando ${ }^{8}$ formando redes de gran tamaño molecular durante el proceso de polimerización asociado a su proceso de "secado" o endurecimiento.

Por tanto, en cualquier caso, el resultado final es que la obra suele estar constituida por sustancias macromoleculares ramificadas y entrecruzadas que, una vez endurecidas y envejecidas, suelen presentar una escasa solubilidad, tanto en agua, como en los disolventes orgánicos clasificados como disolventes débiles (esencia de trementina, white spirit). Por esta razón, estos aglutinantes envejecidos son consideradas por los restauradores como de "escasa reversibilidad".

La eliminación selectiva de estas sustancias será tanto más dificil y arriesgada cuanto mayores sean aquellas modificaciones estructurales experimentadas durante el envejecimiento, especialmente si están relacionadas con una disminución de su reversibilidad. La utilización de métodos de limpieza más agresivos, tales como soluciones alcalinas o disolventes polares poco volátiles y de elevada retención, supondría un aumento del riesgo de actuar sobre las capas subyacentes que no se desean eliminar.

Ante esta situación, una posible alternativa sería la utilización de enzimas hidrolíticas que, por su especificidad, permitirian la eliminación selectiva de estas sustancias envejecidas sin que se vean afectadas las capas subyacentes. En este caso, el mecanismo de actuación es un proceso químico de fragmentación de estos materiales insolubles que son transformados en sustancias de menor tamaño molecular que pueden llegar a ser solubles en el medio acuoso en el que se utilizan las enzimas, con lo que se facilita su eliminación.

Las primeras experiencias relacionadas con la utilización de enzimas en procesos de restauración se realizaron en los años sesenta (Sheridan, 1962). Las enzimas utilizadas en aquellas actuaciones fueron diversas hidrolasas (amilasas, lipasas y proteasas), que se emplearon con la finalidad de eliminar selectivamente algunos materiales que alteraban el estado de las obras.

Hasta el momento, los ejemplos de aplicación práctica de tratamientos enzimáticos en el campo de la restauración constituyen casos aislados y muchas de las experiencias se han llevado a cabo sobre documento gráfico; sin embargo, los resultados han sido positivos y han demostrado que esta nueva metodología puede llegar a ser una alternativa interesante a los tratamientos tradicionales de limpieza de policromías y una práctica habitual en el taller de restauración.
A continuación se describen los aspectos de mayor relevancia para la correcta utilización de enzimas en la restauración de obras de arte; todos ellos han sido tratados, de forma más o menos completa, por los diferentes autores revisados.

\section{Elección de la enzima}

Lógicamente, la elección de la enzima estará determinada por la composición del material que se pretende eliminar, así como por la del resto de los componentes de la obra que deben mantenerse inalterados (Cremonesi, 1997:102, Cremonesi, 2002:46). Con estos datos se puede establecer el tipo de enzima hidrolítica necesaria para resolver cada problema concreto (amilasa, lipasa o proteasa). Una vez seleccionado el tipo de enzima, se debe buscar entre aquéllas que actúen en unas condiciones operativas compatibles con las características de la obra en cuestión $(\mathrm{pH}$, temperatura, tiempo y modalidad de aplicación), lo que a su vez determinará el tipo específico de enzima (Cremonesi, 2002:48-54). Por ejemplo, en el caso de que el tratamiento de limpieza se lleve a cabo sobre una película de óleo, no serían adecuadas aquellas enzimas que actúen a unos valores de $\mathrm{pH}$ superiores a 8,5, ya que estas soluciones podrian provocar procesos de saponificación similares a los que se desarrollan cuando se emplean disoluciones alcalinas.

Respecto al conocimiento de los materiales de la obra, ya se ha mencionado que, además de determinar la naturaleza de los mismos, sería conveniente conocer las alteraciones que han sufrido debido a los procesos de envejecimiento.

El análisis estratigráfico acompañado de técnicas de tinción específicas puede ser un punto de partida para posteriores análisis más exactos, que permitan la identificación de las distintas capas que componen la policromía como sustancias de naturaleza lipídica, proteica o polisacárida. Cremonesi propone un método empírico de determinación de la naturaleza de los aglutinantes que componen la obra, basado en su comportamiento frente a distintos disolventes orgánicos y reactivos químicos (Cremonesi, 2002:48). Este procedimiento podría simplificar la metodología de trabajo y hacerla asequible a los talleres de restauración que no posean las técnicas instrumentales necesarias para el análisis de aglutinantes.

\section{Actividad enzimática y concentración de las disoluciones enzimáticas}

Las enzimas son extraídas de sus fuentes biológicas (bacterias, hongos, tejidos animales o tejidos vegetales), generalmente, mediante los procedimientos típicos de aislamiento y purificación de proteinas. Cualquiera que sea el método empleado, las enzimas se 


\section{Criterios}

Fundamentos y

antecedentes de la

utilización de enzimas en

tratamientos de limpieza

\section{Isabel Blasco Castiñeyra} $\mathrm{y}$ otros

caracterizan por su actividad o capacidad para catalizar una cierta reacción. Incluso cuando las enzimas se utilizan en condiciones óptimas de $\mathrm{pH}$ y temperatura, la velocidad de reacción va a depender de la actividad de la solución enzimática.

La actividad especifica de una enzima se define como el número de unidades de actividad por miligramo (mg) de proteína para un determinado estado de purificación de la proteina (Copeland, 2000:257). A su vez, por convenio se define una unidad de actividad enzimática como la cantidad de enzima que transforma un micromol ( $10^{-6}$ moles) de sustrato por minuto, a una temperatura de $25^{\circ} \mathrm{C}$ y una presión de una atmósfera. Cuando este criterio de definición no se sigue, el fabricante suele especificar las condiciones en las que se han realizado los ensayos de actividad.

Wolbers (1990:112) y Belluchi y Cremonesi (1994) apuntan que para la mayor parte de las proteinas existe un límite de solubilidad próximo a los $10 \mathrm{mg} / \mathrm{ml}$, de forma que cuando se preparan disoluciones que superan esta concentración, el exceso no se disuelve y, por tanto, tampoco contribuye a aumentar la actividad de la solución. Por esta razón, es recomendable elegir enzimas con una actividad especifica elevada para conseguir soluciones efectivas a bajas concentraciones de enzima. Wolbers propone como punto de partida la utilización de soluciones de aproximadamente 1.000 unidades de actividad enzimática/ml (Wolbers 1990:112).

Respecto a los valores de actividad aportados por el fabricante, hay que tener en cuenta que suelen referirse a la actividad de la enzima sobre sustratos diferentes a los que se van a tratar en los procesos de restauración. Por ejemplo, la medida de la actividad de la colagenasa, enzima que cataliza la hidrólisis del colágeno, se realiza sobre el colágeno nativo y no sobre la cola animal empleada en las obras de arte. Aunque la cola se obtiene a partir del colágeno contenido en las pieles, tendones y huesos de vertebrados superiores, su estructura difiere de la del colágeno nativo. Por otra parte, en estas medidas tampoco han sido consideradas las transformaciones que el sustrato haya podido sufrir durante el envejecimiento, ni las posibles interferencias con otros materiales presentes en la obra como, por ejemplo, pigmentos y cargas. Por este motivo siempre será necesario buscar las condiciones más adecuadas a cada caso concreto y realizar ensayos previos sobre los materiales que se van a eliminar (Blasco et al., 2004).

\section{Pureza de las preparaciones enzimáticas}

Para que el tratamiento sea adecuado es necesario conocer con detalle el grado de pureza de la enzima seleccionada. Las enzimas disponibles comercialmente tienen composiciones variables, puesto que a menudo son mezclas; y por otra parte su especificidad y actividad pueden no ser del todo conocidas. En este sentido, hay que señalar que algunas casas comerciales añaden sustancias como estabilizadores o tampones (De Santis, 1983); asimismo,

\footnotetext{
${ }^{9}$ Zimógenos: precursores enzimáticamente inactivos

${ }^{10}$ Tris base: tris (hidroximetil) amino metano
}

otros preparados pueden ser mezclas de varias enzimas o incluso llevar pequeñas proporciones de enzimas no especificadas por el fabricante, que pueden suponer un peligro para la obra tratada. Por ejemplo, algunas proteasas obtenidas a partir de extractos de tejido pancreático pueden ir acompañadas de pequeñas cantidades de lipasas y amilasas. Además pueden contener precursores o zimógenos $^{9}$ de otras enzimas que pueden ser activados por la acción de otras proteasas. Por todas estas razones la utilización de este tipo de preparados no es aconsejable (Belluchi y Cremonesi, 1994).

Dado que la seguridad del tratamiento enzimático dependerá de la pureza de la preparación enzimática utilizada, algunos autores consideran que es importante determinar el grado de pureza y la actividad de una enzima, a partir de la realización de los correspondientes ensayos de actividad (Grattan et al., 1987). A este respecto es importante destacar que para que una enzima pueda ser utilizada en restauración debe tratarse de un reactivo bien caracterizado, con el fin de minimizar el tiempo de actuación y la concentración de la enzima.

\section{Condiciones óptimas de trabajo}

Una vez seleccionada la enzima, es necesario definir las condiciones de trabajo; es decir, pH del medio, temperatura, tiempo de aplicación, concentración de la enzima y presencia de aditivos, ya que éstas resultan fundamentales, tanto para controlar la actividad de la enzima como para evitar posibles daños a la obra tratada.

La mayoría de los autores (Segal and Cooper, 1987; Grattan et al., 1987; Meyer, 1992; Belluchi y Cremonesi, 1994; Cremonesi, 1997:102-104; Cremonesi, 2002:49; Wolbers, 1990:111) consideran que es necesario utilizar las enzimas en sus condiciones operativas óptimas y, además, aconsejan emplear la menor concentración posible de enzima. Esta última recomendación tiene por finalidad minimizar la presencia de residuos enzimáticos que pueden quedar retenidos en la obra después del tratamiento. Este es otro de los factores importantes a considerar y será discutido más adelante.

\section{Medio de aplicación. Utilización de tampones}

Para que la enzima sea activa es necesario trabajar en un medio acuoso que permita que la proteína adopte su conformación nativa. Por esta razón, el agua utilizada debe ser destilada y estar libre de iones metálicos que puedan modificar su actividad. Asimismo, y con el fin de asegurar un valor de pH que permita la acción hidrolítica de la enzima, se suele recurrir a la utilización de disoluciones tampón que, como es sabido, tienen la capacidad de mantener un pH constante, aunque la disolución se ponga en contacto con pequeñas cantidades de ácido o base. En este sentido, es frecuente encontrar sustancias de carácter ácido o básico dentro de la obra, bien debido a su propia composición inicial o a los procesos de envejecimiento. Por ejemplo, los aceites secantes y las resinas naturales tienen un cierto carácter ácido que se puede incrementar durante el envejecimiento. 
Autores como Belluchi y Cremonesi (1994) recomiendan la utilización de tampones que simulen las condiciones biológicas, preparados a una concentración $0,05 \mathrm{M}$ en soluciones acuosas y con un $\mathrm{pH}$ que se ajuste a las necesidades de la enzima elegida.

Aunque los tampones propuestos por los diferentes autores son variados, Cremonesi (2002) aconseja el uso de tampones Tris- $\mathrm{HCl}^{10}$, para valores de $\mathrm{pH}$ comprendidos entre 7,20 y 9,00, y de tampones acetato ( $\mathrm{pH}$ entre 4-5) o tampones fosfato ( $\mathrm{pH}$ entre 6-7) cuando se necesita un pH más bajo.

Por otra parte, ante la necesidad de minimizar la presencia de residuos enzimáticos sobre la obra después de los tratamientos, Diana Nikolova (1994) propone la utilización de tampón acetato 0,1 M a pH 5 $\left(\mathrm{CH}_{3} \mathrm{COONH} \mathrm{CH}_{4} / \mathrm{CH}_{3} \mathrm{COOH}\right)$ debido a la volatilidad de sus componentes.

Hay que destacar que algunos investigadores (Cooper et al., 1987) han utilizado disoluciones acuosas de disolventes orgánicos, concretamente soluciones de 2-metoxietanol o de 2-propanol al $45 \%$ en agua destilada. Estas condiciones han resultado eficaces en la eliminación de restos de almidón mediante el uso de una enzima especifica para fragmentar este tipo de sustancias ( $\alpha$-amilasa); este proceso ha sido llevado a cabo en documentos con tintas solubles en agua que no podian tratarse en un medio totalmente acuoso. Estos autores observaron que, aunque en estas condiciones la actividad de la enzima decaía considerablemente, seguía siendo suficiente para hidrolizar el adhesivo de almidón y favorecer su eliminación.

\section{Temperatura y $\mathrm{pH}$}

El efecto de estos factores sobre la actividad enzimática presenta unas características especiales. En términos generales, cuando la temperatura aumenta, la actividad enzimática también lo hace, hasta alcanzar un máximo, a partir del cual la actividad va decreciendo, debido a la desnaturalización de la enzima (Wolbers, 2000:132-134; Blasco et al., 2004). Un comportamiento semejante se observa si se compara el efecto del pH sobre la actividad enzimática; existe un valor de pH óptimo para el que la actividad de la enzima es máxima, y por debajo o por encima de este valor, la actividad enzimática es menor (Blasco et. al., 2004) . Dependiendo de la enzima, el intervalo de $\mathrm{pH}$ o temperatura en el que la actividad es suficientemente elevada es más o menos amplio (Gráfico 1). Lógicamente, en aquellas enzimas que presentan un intervalo muy pequeño de $\mathrm{pH}$ o temperatura óptimos, el control de estos parámetros debe ser más riguroso (Cremonesi, 1997:49-50).

Una modificación de pH puede producir un cambio conformacional de la enzima que podría llegar a afectar a la región donde se encuentra el centro activo, variando asi la accesibilidad del sustrato a dicho centro. Por otra parte, el pH va a condicionar el estado de ionización de ciertos grupos funcionales que son fundamentales en el mecanismo de actuación de la enzima.
Wolbers ha comprobado que, cambiando las condiciones de $\mathrm{pH}$, una misma enzima puede mostrar una mayor afinidad por un tipo $u$ otro de sustrato. Experimentalmente, este mismo autor comprueba que el pH 8,5 es el adecuado para la hidrólisis de aceite de linaza utilizando una lipasa (Wolbers, 1990:113).

Cuando se utilizan disoluciones acuosas de disolventes orgánicos como medio de aplicación, Cooper y sus colaboradores (1987) destacan la importancia del mantenimiento del pH óptimo, que en este caso es, además, distinto del recomendado para medios acuosos. Concretamente, para la $\alpha$ - amilasa preparada en 2 -metoxietanol (al $45 \%$ en agua destilada), observan que un cambio de $\mathrm{pH}$ de 7 a 7,2 es responsable de que la actividad enzimática se vea reducida a la mitad.

Accesibilidad del sustrato

En general, las reacciones catalizadas por enzimas en los organismos vivos son reacciones en fase homogénea; es decir, el sustrato está disuelto en el medio acuoso en el que se encuentra la enzima. Por el contrario, en la obra de arte, el sustrato es una superficie sólida, insoluble en el medio acuoso en el que se disuelve la enzima, de forma que ésta tiene que difundirse previamente en el sustrato para poder actuar sobre él. Esta circunstancia hace que la reacción sea más difícil o, al menos, más lenta (Cremonesi, 2002:56).

El poder de difusión de la enzima se verá limitado por el gran tamaño que éstas suelen presentar en disolución. Este hecho se complica más si la naturaleza de la superficie es hidrofóbica, como ocurre en el caso de las películas de óleo (Wolbers, 1990a:111; Belluchi y Cremonesi, 1994) o en superficies que presentan una capa de suciedad de carácter graso, ya que la superficie externa que muestra la conformación de la enzima en medio acuoso es fundamentalmente hidrofílica.

Para reducir este problema, muchos autores han optado por la utilización de sustancias tensoactivas (en concentraciones muy bajas) que mejoran el mojado de la solución enzimática y, de esta manera, se favorece la difusión y se facilita la accesibilidad de la enzima al sustrato, promoviendo la reacción enzimática. Wolbers propone la adición de tensoactivos no iónicos como el Triton X-100 (Wolbers, 1990:111) o la utilización del ácido deoxicólico (Wolbers 2000:136). A su vez, Belluchi y Cremonesi (1994) proponen utilizar otros tensoactivos no iónicos como la bilis de buey, destacando que este tipo de tensoactivos no alteran el equilibrio electrolítico y, por tanto, la solubilidad de la enzima.

No obstante, siempre que se añaden sustancias a la solución enzimática hay que tener en cuenta las posibles interferencias en su actividad (Knox, 1995).

\section{Utilización de agentes espesantes}

Los primeros tratamientos de limpieza con enzimas fueron realizados sobre obra gráfica, y en estas primeras experiencias, el método de apli- 


\section{Criterios \\ Fundamentos y \\ antecedentes de la \\ utilización de enzimas en \\ tratamientos de limpieza \\ Isabel Blasco Castiñeyra \\ y otros}

cación consistía en la inmersión de la obra en la solución enzimática. Esta metodología implicaba la necesidad de preparar grandes volúmenes de este tipo de soluciones y además resultaba problemática cuando las tintas o las policromías eran solubles en medio acuoso. La solución que se propuso a este problema fue el uso de geles preparados a partir de disoluciones coloidales de elevada viscosidad, conocidas también como espesantes, en las que a su vez se disolvía la enzima.

La primera cita relacionada con la utilización de estas sustancias como sistema para la aplicación local de soluciones enzimáticas ya mencionaba las posibles ventajas de este método (Hatton, 1977), que son: necesidad de un menor volumen de disolución enzimática (con lo que se reducen costes), posibilidad de aplicación puntual en la obra de arte y además transparencia de los geles, propiedad que permite observar la evolución del proceso.

Otra ventaja asociada al uso de estas soluciones es que disminuyen la penetración, lo que es importante cuando la película pictórica está fragmentada (Wolbers, 1988). En un trabajo posterior se resalta la ventaja de mantener las condiciones de humedad durante un tiempo más prolongado (Blüher and Banik, 1996).

Los agentes espesantes más recomendados son los éteres de celulosa y, entre ellos, los más comunes son: la metilcelulosa (comercializada con distintas denominaciones según el fabricante: Benecel, Culminal, Glutofix, Methocel A, Tylose) y la hidroxipropilcelulosa (de nombre comercial Klucel), todos ellos compuestos que en disolución acuosa se comportan como no electrolitos y, por tanto, son compatibles con todas las condiciones de pH (Cremonesi, 2000:52). Por el contrario, la carboximetil-celulosa, al ser una sal sódica, en disolución acuosa se comporta como un polielectrolito por lo que su acción espesante se ve afectada por las condiciones de $\mathrm{pH}$; concretamente cuando el medio es ácido su acción espesante disminuye, por lo que en estas condiciones no es aconsejable su utilización.

La viscosidad recomendada para este tipo de soluciones es de $4000 \mathrm{cp}$ (centipoises), valor que se alcanza con concentraciones entre 2-4\% peso/volumen (Cremonesi 2002:52).

El principal inconveniente asociado al empleo de agentes espesantes es la posible permanencia de residuos sólidos en la obra, lo que se puede reducir con un lavado adecuado. Otro aspecto que hay que considerar es su efecto sobre la actividad de la enzima, que normalmente se ve modificada y más concretamente disminuida; en este sentido, Erickson (1992) aconseja un incremento de la concentración de enzima en un factor de orden 10, con respecto a la utilizada en soluciones acuosas.

\section{Residuos enzimáticos}

Uno de los principales problemas que surge, como consecuencia de la utilización de enzimas en procesos de limpieza de obras de arte, es la posible permanencia de residuos después del tratamiento, y la falta de un conocimiento preciso sobre el efecto negativo que éstos pueden llegar a provocar sobre la obra a largo plazo. Para reducir la actividad de los posibles residuos enzimáticos, una vez finalizado el tratamiento de limpieza, el procedimiento general aconsejable es el siguiente:

> Lavado de la zona tratada para eliminar al máximo los restos de componentes de la solución enzimática: enzimas, tampones, agentes espesantes y tensoactivos.

> Desactivación de la enzima con el fin de asegurar que los posibles residuos no sean activos.

Como métodos de lavado se han utilizado diferentes alternativas: agua destilada, tampón de la disolución enzimática empleada (Meyer et al., 1992), saliva sintética (Cremonesi, 2002:86) y disolventes orgánicos (Tallent, 1985).

Para conocer la eficacia de estos procedimientos, se recomienda cuantificar los residuos una vez efectuados los distintos tratamientos para su eliminación. Algunos autores han desarrollado protocolos que permiten valorar cuantitativamente estos residuos (TaIlent, 1985, Erickson, 1992, Meyer et al., 1992).

Las primeras medidas de residuos enzimáticos fueron realizadas después del tratamiento con una lipasa. La cantidad de enzima eliminada después de los lavados se determinó mediante un método colorimétrico de detección de proteínas (Tallent, 1985).

Meyer et al., (1992) realizaron un estudio utilizando enzimas marcadas con yodo radiactivo sobre papel evaluando, por una parte, la eficacia de distintos procedimientos de lavado y, por otra, la influencia de las condiciones de realización de la incubación ${ }^{11}$ en la cantidad de enzima retenida. Las principales conclusiones de los resultados obtenidos fueron las siguientes:

> La cantidad de enzima retenida en el papel no era muy elevada y, aparentemente, dependía de la pureza y concentración de la enzima. Ésta se incrementaba al aumentar su concentración, lo que confirma la necesidad de utilizar las concentraciones más bajas posibles en los tratamientos, partiendo de preparados enzimáticos de máxima pureza.

> La utilización como sistema de lavado de soluciones con el pH óptimo, recomendado para las enzimas durante el tratamiento enzimático, daba lugar a una ligera reducción en la cantidad de enzima retenida. 
Los autores destacan que estos resultados no pueden generalizarse para otras enzimas distintas a las ensayadas y sugieren la realización de estudios al respecto.

Por otra parte, para lograr la desactivación de la enzima se puede optar por su desnaturalización o por su inhibición. En el primer caso se han propuesto distintas alternativas: a) la realización de lavados con agua caliente (Hatton, 1977), b) la utilización de disolventes (Segal and Cooper, 1977, Hatton, 1977) o c) la modificación del pH. En cualquier caso, es absolutamente necesario efectuar un lavado previo con el fin de eliminar la mayor cantidad posible de residuo enzimático, puesto que la desnaturalización puede provocar su precipitación (De Santis, 1983; Erickson, 1992).

En cuanto a la utilización de inhibidores enzimáticos, Nikolova (1993) propone la posibilidad de utilizar estos inhibidores para evitar posibles situaciones de riesgo, después de la realización de tratamientos enzimáticos. Las principales ventajas que presenta su utilización serian: gran especificidad, rapidez de actuación, fácil disolución en agua y escasa peligrosidad en su manipulación. No obstante, las investigaciones realizadas no han sido suficientes y queda por establecer la eficacia de estos tratamientos y sus posibles efectos sobre la obra de arte.

\section{Conclusiones}

$>$ Los sistemas tradicionales de limpieza de policromías son, a menudo, poco específicos y agresivos para la obra y tóxicos para el restaurador. Frente a éstos, las enzimas presentan como principales ventajas: a) especificidad de actuación sobre los materiales que se quieren eliminar sin que se vean afectados otros componentes de la obra, b) eficacia de los tratamientos en unas condiciones de trabajo poco agresivas para la obra de arte y c) baja toxicidad para el restaurador.

$>$ Las enzimas que resultan eficaces para su uso en procesos de limpieza de obras de arte son las hidrolasas: amilasas, proteasas y lipasas, que fragmentan respectivamente el almidón, las proteinas y los lípidos. Su elección dependerá de la naturaleza de la sustancia que se quiera fragmentar y de las características de los materiales que deben permanecer inalterados.

> Para su empleo hay que tener en cuenta las características de la enzima (actividad y pureza), así como las condiciones óptimas de trabajo (concentración, pH, temperatura, tiempo de actuación). Además, estas condiciones deben ser compatibles con las características de la obra que se va a tratar.

> Para minimizar los posibles residuos enzimáticos que puedan quedar sobre la obra una vez efectuado el tratamiento, es nece- sario realizar los correspondientes lavados. También se recomienda su desactivación que debe realizarse siempre después del proceso de lavado.

$>$ Los trabajos realizados sobre el empleo de enzimas en el campo de la restauración son escasos y han sido realizados principalmente sobre obra gráfica. Además las publicaciones derivadas de estas investigaciones no suelen detallar los protocolos seguidos, por lo que en muchos casos, su interpretación resulta difícil.

> Sería necesario desarrollar una metodología específica para estudiar la eficacia de estos tratamientos en la limpieza de obras policromadas. Como fase previa es imprescindible realizar la elección y caracterización de las enzimas más adecuadas para eliminar las distintas sustancias que alteran la obra de arte (suciedad, repintes, sustancias alteradas), así como el estudio de su especificidad, eficacia y condiciones óptimas de utilización.

> Debido a las características propias de la obra, la retención específica de la enzima y la complejidad de las disoluciones empleadas, sería muy aconsejable la cuantificación de los residuos después de los tratamientos y estudiar su efecto a largo plazo.

\section{Agradecimientos}

Este trabajo forma parte del proyecto (06/0171/03) titulado "La utilización de enzimas en los procesos de limpieza de obras de arte" financiado por la DGI de la Consejería de Educación de la Comunidad de Madrid. Las autoras también agradecen a Silvia García Fernández-Villa la realización de las figuras.

\section{Bibliografía}

BELLUCHI, R. Y CREMONESI, P. (1994). L' uso degli enzymi nella conservazione e nel restauro dei dipinti. Kermes, $n^{\circ} 21$, p. 45-64

BLASCO, I., VIÑA FERRER, S., SAN ANDRÉS, M. (2004). La utilización de enzimas proteolíticas en procesos de limpieza de policromías. Importancia de la realización de estudios previos de caracterización de los preparados enzimáticos". XV Congreso de Conservación y Restauración de Bienes Culturales. Murcia, del 21 al 24 de octubre de 2004. Pendiente de publicación

BURNSTOCK, A Y WHITE, R. (1990). The effects of selected solvents and soaps on a simulated canvas painting in Mills, J.s and Smith, P., Preprints of the Contributions to the Brussels Congress. International Institute for Conservation of Historic and Artistic Works. Londres, p. 111-118

BURNSTOCK, A Y LEARNER, T. (1992). Changes in the surface characteristics of artificially aged mastic varnishes after cleaning using alkaline reagents. Studies in Conservation 37, n³. p. 165-184

BLÜHER, A., BANIK, G. (1996). The aplication of enzyme-containing methylcellulose gels for the removal of starch-based adhesives in albums. Paris ICOM. p. 494-499 


\section{Criterios}

Fundamentos y

antecedentes de la

utilización de enzimas en

tratamientos de limpieza

Isabel Blasco Castiñeyra

y otros

CALEY, T. (1990). Aspects of varnishes and the cleaning of oil paintings before 1700. Cleanig Retouching and Coatings. Technology and practice for Easel Paintings and Polychrome sculpture. Preprints of the Contributions to the Brussels Congress. International Institute for Conservation of Historic and Artistic Works. Londres, p. 70-72

CARLYLE, L, TOWNSEND, J.H. Y HACKNEY, S. (1990). Triamonium citrate: an investigation into its application for surface cleaning. Dirt and Pictures Separated. Reino Unido, Institute for Conservation of Historic and Artistic Works. p. $44-48$

CREMONESI, P. (1997). Materiali e metodi per la pulitura di opere policroma. BoIonia: Phase, 1997

CREMONESI (2002). L' 'uso degli enzimi nella pulitura di opere policrome. (II edizione). Padova: II Prato, 2002

COPELAND, R.A. (2000). Enzymes. A practical Introduction to structure, Mechanism and data analysis. Nueva York: John Wiley \& Sons, 2000

COOPER, D., KING, C Y SEGAL, J. (1987). The use of enzymes in partially non aqueous methods. Conservation of library and archive materials and the Grafic Arts. Londres: G.Petherbridge, 1987, p. 25-30

DE SANTIS, PIA (1983). Some observations on the use of enzymes in paper conservation. J.A.I.C. Vol. $23, n^{\circ} 1$, p. $7-27$

ERHARDT, D. Y TSANG, J-S. (1990). The extractable componets of oil pain films. Cleaning Retouching and Coatings. Technology and practice for Easel Paintings and Polychrome sculpture. Preprints of the Contributions to the Brussels Congress. International Institute for Conservation of Historic and Artistic Works. Londres, p. 93-97

ERICKSON, H.M. (1992). Usage recommendations for á-amylases: Maximizing Enzyme activity while minimizing enzyme-artifact binding residues. The Book and Paper Group Annual. Vol. 11

FELLER, R.L., STOLLOW, N., Y JONES, E.H (1985). On picture Varnishes and their Solvents. Washinton, DC: National Gallery of Art, 1985

FERSHT, A. (1984). Enzyme structure and mechanism. Nueva York: W.H. Freeman and Company, 1984

FISCHER, E. (1884). Einfluss der configuration auf die wirkung derenzyme. Ber. Dt. Chem. Ges. 27, p. 2895-2993

GRATTAN, D. ST, HILAIRE, J. BURGESS, H. Y MC CAWLEY, C.(1987).The characterization of enzymes for use in paper Conservation. Conservation of library and archive materials and the Grafic Arts.Ed. G.Petherbridge. Londres: Butterworths, p. $15-25$

HATTON, M. (1977). Enzymes in a viscous médium. Paper Conservator 2 (9)

HEDLEY, G., ODLYHA, M, BURNSTOCK, A., TILLINGHAST, J. Y HUSBAND, C. (1990). A study of the mechanical and surface properties of oil paint films treated with organic solvents and water. Cleaning Retouchings and Coatings. Technology and practice for Easel Paintings and Polychrome sculpture. Preprints of the contributions to the Brussels Congress. International Institute for Conservation of Historic and Artistic Works. Londres, p. 98-105

KNOX, S. (1995) An Assessment of catalytic activity in Wolber's lipase gel using an enzyme assay. Conservation News. $n^{\circ} 58, p .47$
KOSHLAND, D.E. JR Y NEET, K.E. (1968) The catalytic and regulatory properties of enzymes. Ann. Rev. Biochem, p. 359-410

LEHNINGER (1985) Bioquimica. Barcelona: Ediciones Omega, 1985

MARCHI MENEGUZZI, R. E. Y OTOGALLI PERRINO, F. (1999). Patologie da solventi per gli addetti al restauro. Padova: Ed. II Prato, 1999

MASSCHELIN-KLEINER, L. (1991). Les Solvants. Bruselas: Institut Royal du Patrimoine Artistique, 1991

MEYER ANDREWS, T, ANDREWS, WW. Y BAKER, C. (1992). An investigation into the removal of enzymes from paper following conservation treatment. JAIC vol. $31, n^{\circ} 3$, p. $313-323$

NIKOLOVA, D. (1993). Proteinase inhibitors from vegetables and their applicaton in enzyme conservation treatments. Restaurator, 14, p. 199-216

NIKOLOVA, D. (1994). Enzymatic separation of oil painting layer in transfer to a new base. News of the National Museum of History, vol X, p. 217220

PHENIX, A Y BURNSTOCK, A. (1990). The deposition of dirt: a review of the literature, with scanning electron microscope studies of dirt on selected paintings. Dirt and Pictures Separated. Reino Unido: Institute for Conservation of Historic and Artistic Works, 1990

PHENIX, A.; BURNSTOCK, A. (1992). The removal of surface dirt on paintings with chelanting agents. The Conservator, 16. P. 28-38

PHENIX A. Y SUTHERLAND, K. (2001). The cleaning of paintings: effects of organic solvents on oil paint films. Reviews in Conservation. The International Institute for Conservation of Historic and Artistic Works (IIC). N ${ }^{\circ} 2$, p. $47-60$

PRICE Y STEVENS (1989). Fundamentals of enzymology, Oxford: Science Publications, 1989

SEGAL, J. Y COOPER, D. (1977). The use of enzymes to release adhesives. Paper Conservator 2 p. $047-50$

SHERIDAN, J. (1962). Enzymes, as they relate to the conservator of paintings. In exposition of painting conservation, Brooklyn Museum

STOLLOW, N. (1957 a). The action of solvents on drying-oil films: part I. J. Oil and Colour Chemists. Assoc. 40, p. 377-402

STOLLOW, N. (1957 b). The action of solvents on drying-oil films: part II. J. Oil and Colour Chemists. Assoc. 40, p. 488-499

TALLENT, C. (advisor Wolbers, R) (1985). Development of a technique to remove oil overpaint using lipase, a lipolytic enzyme. Student science project. University of Delaware. Art Conservation Training Program.

WOLBERS, R. C. (1988). Aspects of the examination and cleaning of two portraits by Richard and William Jennys. AIC $16^{\text {th }}$ Annual Meeting. New Orleans, L.A. p. 245-261

WOLBERS, R. (1990). Notes for workshop on new methods in the cleaning of paintings. Getty Conservation Institute.

WOLBERS, R. (2000). Cleaning painted surfaces. Aqueous Methods. Londres: Archetype Publications, 2000 